\title{
Harris Corner Detection Algorithm at Sub-pixel Level and Its Application Yuanfeng $\mathrm{Han}^{\mathrm{a}}$, Peijiang Chen ${ }^{\mathrm{b}}{ }^{*}$ and Tian Meng ${ }^{\mathrm{c}}$ \\ School of Automobile, Linyi University, Shandong, 276000, China \\ a hyf2012413018@163.com, b *chenpeijiang@163.com, '1257398576@qq.com
}

Keywords: Harris corner, Sub-pixel, Gaussian surface, Image matching

\begin{abstract}
The corner is one of the most important features of image and the corner detection plays an important role in digital image processing technology. The performance of Harris corner detection algorithm is stable and it is widely applied, but the corner location is pixel level which affects the subsequent image processing. In order to improve the performance of corner detection, the harris algorithm is improved. On the basis of analyzing the principle of Harris corner detection algorithm, the sub-pixel level corner position based on the Gauss surface method is extracted. The extracted corners is applied in images matching. The experimental results show that the Harris corner detection algorithm can extract high accurate corners. At the same time, the image matching based on this algorithm has higher precision.
\end{abstract}

\section{Introduction}

The corner reflects the characteristics of image's essence which brightness changes strongly [1]. The real corner can effectively simplifies the information amount of data processing. Then, it significantly improves the speed of image processing and matching accuracy and realizes the efficiency of image processing. Therefore, in the entire image processing process, corner extraction is particularly important. Further, corner extraction is more accurate and more conducive to analyze and process image information [2]. At the same time, corner extraction plays a crucial role in determining the object in the real environment, even it is significant for stereo matching view.

Harris algorithm is a classical corner detection method. Its comprehensive effect is good and it is widely applied, but there are also many limitations. For example, it can be only applied in a single scale, and it is difficult to implement multi-scale corner detection. And the threshold value should be given manually, in addition, the threshold value directly affects the effect of corner extracting. Harris algorithm extracts corner based on pixel level, which has been unable to meet the requirement for precision. With the rapid development of computer technology, the sub-pixel corner detection has become an important part of the computer image processing.

In this project, aiming at the shortcomings of the Harris algorithm, first of all, using Harris detection algorithm extracts the feature points, then, adopting Gaussian surface fitting improves the accuracy of the extracted feature points, which would lay a foundation for the stereo matching of feature points, and it is applied to the image matching.

\section{Harris corner detection}

\section{The algorithms of corner detection}

Over the years, the science researchers have been committed to the corner detection field. The corner detection plays an important role in the field of image processing. At present, the corner detection methods are mainly divided into two categories: based on image edge information and based on image gray scale information [3].

The corner detection in image processing has the following requirements.

(1) Stability. The corner detection algorithm has strong adaptability to gray scale images, lines and brightness, which can make proper adjustment automatically, and the detected corner number and position are stable. 
(2) Resistance for noise. During a certain amount of noise, the algorithm can weaken or eliminate the noise, and it has strong ability of corner detection.

(3) Accuracy. It can find the accurate coordinates of corners.

(4) Efficiency. The method is fast enough and it saves running time.

\section{The principle of Harris corner detection}

Harris algorithm basically meets the requirements of the corner detection, thus it has quite ideal effect, and it is widely applied. It uses a certain threshold to extract the feature points by the analysis of image gray's change. The color image is transformed into a gray image. Differential operator can reflect the change of pixel gray level in any direction, thus effectively distinguish the corners and edge points [4]. The change of gray intensity can be determined by analyzing the moving pixels in any direction.

Harris corner detection is mainly to use gray information to detect corners and its principle is the image gray scale average difference which is caused by the image window deviating in any direction.

The auto-correlation equation is expressed, as shown in Eq. 1.

$$
E(x, y)=\sum_{m, n} w_{m, n}\left|I_{x+m, y+n}-I_{x, y}\right|^{2}
$$

Where, $w$ is the image window function.

$E(x, y)$ can be approximately expressed, as shown in Eq. 2.

$$
E(x, y)=A x^{2}+B y^{2}+2 C x y
$$

Where, $A=X^{2} \otimes w, B=Y^{2} \otimes w, C=X Y \otimes w, w$ can select the Gaussian smoothing function, $X$ and $Y$ are first order directional derivatives.

$$
E(x, y)=\left[\begin{array}{ll}
x & y
\end{array}\right] M\left[\begin{array}{l}
x \\
y
\end{array}\right]=\left[\begin{array}{ll}
x & y
\end{array}\right]\left[\begin{array}{ll}
A & C \\
C & B
\end{array}\right]\left[\begin{array}{l}
x \\
y
\end{array}\right]
$$

Matrix $M$ is approximately the Hessian matrix of autocorrelation function, the judgment function can be defined, as shown in Eq. 4.

$$
R(x, y)=\operatorname{det}[M(x, y)]-k \cdot \operatorname{trace}^{2}[M(x, y)]
$$

Where, $\operatorname{det}()$ is the determinant of the matrix, and trace() is its trace.

If the $R(x, y)$ of a point is greater than certain threshold, it can be made as a corner.

From the algorithm's operation, the threshold setting is a very critical problem. The threshold setting determines the effect of corner extraction, the value of threshold depends on the human experience [5]. If the threshold is too large, it is easy to lose corners, and if the threshold is too small, it is easy to extract false corners. The threshold setting greatly depends on the nature of the real image, especially when the image color changes, the threshold is more difficult to choose [6].

In addition, there are some problems such as the detected corners are pixel level, which affects the following accuracy of image processing.

\section{The Sub-pixel corner detection of Harris algorithm}

The Harris algorithm extracts corners at pixel level, the positioning precision is low. In order to improve the precision of Harris corner, the sub-pixel detection methods are used [7]. Now, these methods are mainly divided into three categories: interpolation method, moment method and fitting method.

The fitting method is widely used in the field of digital image processing. There are various kinds of surface fitting, such as Gaussian surface fitting, polynomial fitting method, ellipse fitting method. This project focuses on the Gaussian surface fitting algorithm, which can realize the positioning precision at level of the sub-pixel.

First of all, using the Harris operator can carry out coarse position of corners at pixel level. Then, the coarse positioning pixel is made as the center. The appropriate window is selected to get the 
corresponding information of grey value. Finally, the corners at the level of sub-pixel can be gotten, which can improve the extraction precision of image corners [8].

The equation of Gaussian surface can be expressed, as shown in Eq. 5.

$$
z=\frac{n}{2 \pi \sigma^{2}} e^{\frac{-\rho^{2}}{2 \sigma^{2}}}
$$

Where, $\rho=\sqrt{\left(x-x_{0}\right)^{2}+\left(y-y_{0}\right)^{2}}$

Take the logarithm on both sides.

$$
\begin{aligned}
\ln z & =-\frac{x^{2}}{2 \sigma^{2}}+\frac{x \times x_{0}}{\sigma^{2}}-\frac{y^{2}}{2 \sigma^{2}}+\frac{y \times y_{0}}{\sigma^{2}}+n_{0} \\
& =n_{0}+n_{1} x+n_{2} y+n_{3} x^{2}+n_{3} y^{2}
\end{aligned}
$$

Where, $n_{0}, n_{1}, n_{2}, n_{3}$ are undetermined parameters, $n_{0}=\ln (n)-\ln \left(2 \pi \sigma^{2}\right)-\frac{x_{0}{ }^{2}}{2 \sigma^{2}}-\frac{y_{0}{ }^{2}}{2 \sigma^{2}}, n_{1}=\frac{x_{0}}{\sigma^{2}}$, $n_{2}=\frac{y_{0}}{\sigma^{2}}, n_{3}=-\frac{1}{2 \sigma^{2}}$.

According to the least squares principle, the parameters can be gotten, and the corner position can be obtained, as shown in Eq. 7.

$$
x_{0}=-\frac{n_{1}}{2 n_{3}}, \quad y_{0}=-\frac{n_{2}}{2 n_{3}}, \quad \sigma=\sqrt{-\frac{1}{2 n_{3}}}, \quad n=-\frac{\pi}{n_{3}} e^{n_{0}-\frac{n_{1}^{2}}{4 n_{3}}-\frac{n_{2}^{2}}{4 n_{3}}}
$$

Then, $\left(x_{0}, y_{0}\right)$ is the Gaussian surface extreme value point position.

In the project, the corners of two automobile image are detected by Harris algorithm combining with the Gaussian surface method, as shown in Fig 1. The false corners are little and they have high precision.

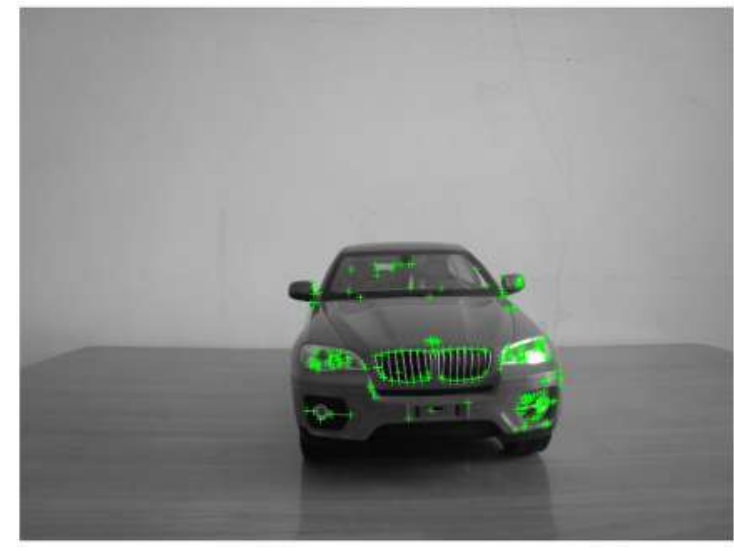

(a) Left image

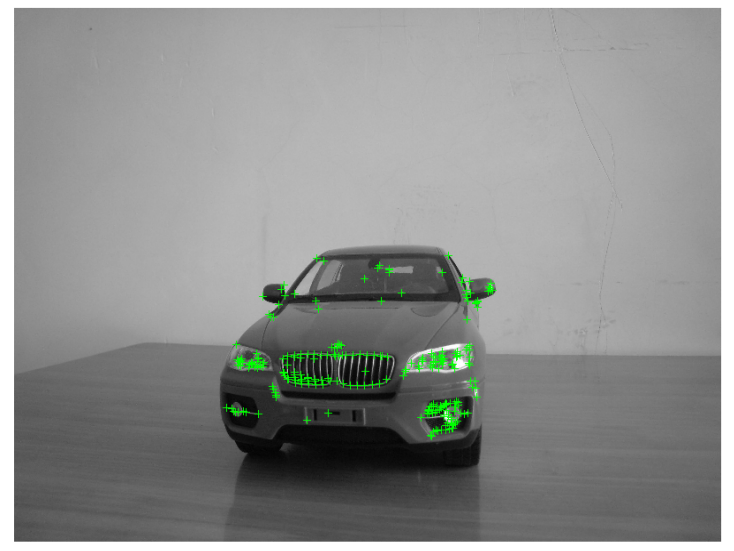

(b) Right image

Fig. 1 The detected corners of two automobiles

\section{Image matching based on Harris corners}

Image matching is the process of looking for similar image or sub-image in an image with a given target image [9]. The technology belongs to the key content in the field of computer vision.

Due to uncontrollable factors, such as noise, light wave interference, the feature points on the two cameras of the same calibration object will be different. In addition, the feature points on left and right image may be no longer the mapping relationship of one-to-one. A feature on the left imaging plane may correspond to several characteristics on the right imaging plane.

In order to make image matching more accurate, it is usually to set a variety of constraints on them. 
(1) The candidate matching based on correlation theory

Usually, making a feature point of the image as the center, a sub-image window is selected as the neighborhood window of the feature point. The similarity would be compared between the left neighborhood window of a given feature point in the left image and each right neighborhood window of feature point in the right image. According to the normalized formula of correlation coefficient at gray-level, the calculation of similarity can be described, as shown in Eq. 8.

$$
S(x, y)=\frac{\sum_{y^{\prime}=0}^{n-1} \sum_{x^{\prime}=0}^{m-1}\left(T\left(x^{\prime}, y^{\prime}\right)-\bar{T}\right)\left(I\left(x+x^{\prime}, y+y^{\prime}\right)-\bar{I}\right)}{\sqrt{\sum_{y^{\prime}=0}^{n-1} \sum_{x^{\prime}=0}^{m-1}\left(T\left(x^{\prime}, y^{\prime}\right)-\bar{T}\right)^{2} \sum_{y^{\prime}=0}^{n-1} \sum_{x^{\prime}=1}^{m-1}\left(I\left(x+x^{\prime}, y+y^{\prime}\right)-\bar{I}\right)^{2}}}
$$

Where, $S(x, y)$ is the similarity of two corresponding neighborhood window, $I$ is gray scale value of target image, $\bar{I}$ is the average gray scale value of neighborhood window in the target image; $T$ is the gray scale value of source image, $\bar{T}$ is average gray scale value of neighborhood window in the source image.

(2) Matching strength calculation of corresponding points based on disparity gradient

Candidate matching based on correlation means are more concentrated. Because of the brightness and environmental conditions, a certain feature point in one image may have many similar points in another image. The disparity gradient can be used to detect the consistency degree of matched points [10].

(3) Calculating the fundamental matrix based on candidate matched points

The linearity of fundamental matrix can be estimated by using the normalized coordinates of corresponding points. Before the calculation, the corresponding coordinates of feature points can be processed by normalization. The normalization matrix is obtained by using least square method. After the adjustment based on rank-2 constraint, the fundamental matrix can be gotten.

According to the fundamental matrix, it can eliminate most false matching, so the matched corners has high registration precision.

For the tow automobile images as shown in Fig. 1, the detected Harris corners are matched based on the above method, the corner matching results are given, as shown in Fig. 2. The matching result of the detected Harris corners has higher accuracy.

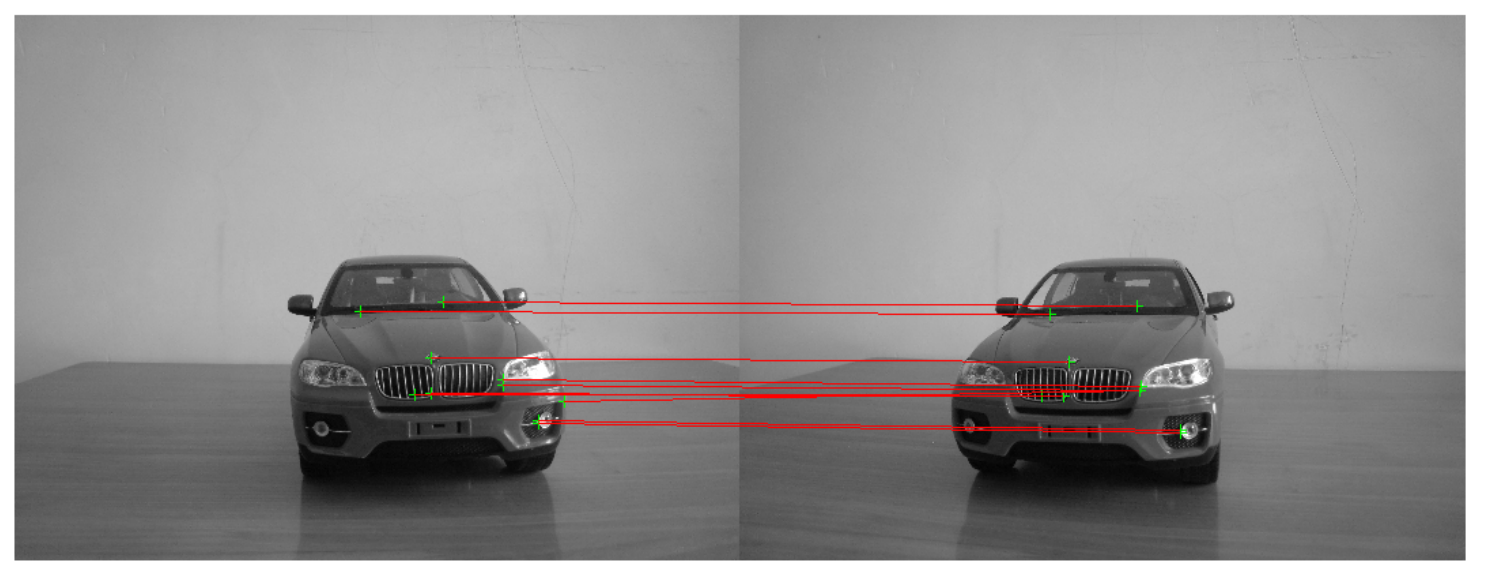

Fig. 2 The corner matching results of the two automobile images

\section{Conclusions}

On the basis of Harris algorithm, the Gaussian surface fitting is used to extract the the sub-pixel corners in this project. The experimental results show that extracted corners are more accurate, and the experiments of image matching can also verify the effectiveness. It can be used in some 
applications with higher demand of positioning precision. Of course, there are also something need to be improved. For example, the Harris algorithm uses certain threshold to detect the corners, which affects the universality of the algorithm may bring certain difficulty for subsequent precision positioning. The related research needs to be continued to improve the practicability of the corner detection method.

\section{Acknowledgements}

This study is sponsored by the National Undergraduate Training Programs for Innovation and Entrepreneurship (No. 201410452031) and the Science and Technology Development Planning Project of Shandong Province (No. 2013YD05004), People's Republic of China.

\section{References}

[1] F. Bellavia, D. Tegolo, C. Valenti, Improving Harris Corner Selection Strategy. IEF Computer Vision. 5(2) (2011) 87-96.

[2] Y. Bastanlar, Y. Yardimci, Corner Validation Based On Extracted Comer Properties. Computer Vision and Image Understanding. 5(3) (2008) 112-114.

[3] W. Zhao, S. Gong, C. Liu, X. Shen, Adaptive Harris Corner Detection Algorithm. Computer Engineering. 34(10) (2008) 212-215.

[4] X. Wang, Z. Wang, F. Wu, Harris Correlation and Feature Matching. Pattern Recognition and Artificial Intelligence. 22(4) (2009) 505 -513.

[5] D. G. Lowe, Distinctive Image Features From Scale-Invariant Key-Points. International Journal of Computer Vision. 60(2) (2004) 91-110.

[6] Y. Li, R. Xia, Sub-pixel Edge Detection Algorithm Based on Curve Fitting Method. Transaction of Chongqing College of Technology. (10) (2008) 82 -84.

[7] Z. M. Liang, H. M. Gao, Z. J. Wang, L. Wu, Sub-pixels Corner Detection for Camera Calibration. Transctions-China Welding Institution. 27(2) (2006) 102-104.

[8] D. Zhao, D. Liu, Y. Yang, A New Stereo Matching Method Based On Sub-pixel Corner Detection. International Conference on Computer Science and Software Engineering. (2) (2008) 899-902.

[9] Q. Zhu, B. Wu, N. Wan, A Sub-pixel Location Method for Interest Points By Means of the Harris Interest Strength. The Photogrammetric Record. 22(120) (2007) 321-335.

[10] A. Trujillo-Pino, K. Krissian, M. Aleman-Flores, D. Santana-Cedres, Accurate Sub-pixel Edge Location Based On Partial Area Effect. Image and Vision Computing. 31(1) (2013) 72-90. 